\title{
COMPUTER ASSISTED DYNAMIC NAVIGATION SYSTEMS -CURRENT SCENARIO
}

\author{
Dr. V. R. Balaji, Dr. D. Manikandan, Dr, Dr. G. Rohini, Dr. M. Nivethitha
}

Department of Periodontology and Implantology, CSI College of Dental Sciences and Research, Madurai, Tamilnadu, India

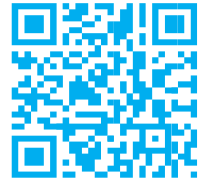

DOI: 10.37841/jidam_2021_V8_I3_03

Address for Correspondence

Dr. M. Nivethitha,

Postgraduate student,

Department of Periodontology and Implantology,

CSI College of Dental Sciences and Research,

Madurai. Tamilnadu, India

E-mail id: dpmnivi@gmail.com

Received: 14.08.2021

First Published: 07.09.2021

Accepted: 02.09.2021

Published: 27.09.2021

\section{ABSTRACT}

One of the techniques available for prosthetic rehabilitation of lost teeth are implants. Earlier implants were placed in a surgically driven treatment approach. Later the idea of placing implants on the basis of prosthetically driven treatment approach was derived by introduction of computer assisted surgery. Dynamic navigation is a technique of computer assisted surgery where the operator can perform accurate, easy and flapless implant insertion surgeries even within a single day. These navigation systems are continuously evolving to achieve even better ways of implant placement. This review will briefly highlight on the details of this navigation implant surgery.

KEY WORDS: Dynamic navigation, Static system, Trace registration, Evalunav. 


\section{INTRODUCTION}

The treatment modalities available for replacement of missing teeth have evolved over the last centuries. Earlier, implants were intended to be placed in areas with greatest amount of bone within the arch, with less regards to the placement of final definitive restoration thus causing failures. In order to cater to this requirement, CGISComputer Guided Implant Surgery and CNIS- Computer Navigated Implant Surgery were introduced. Dynamic navigation (DN) seems to be a tool for navigation thus improving the accuracy of implant placement.

\section{HISTORICAL PERSPECTIVE}

Various software using Computerized tomography were available in late 1980 's. Initially in 1988, the Columbia scientific INC., developed a software to convert the axial slices of CBCT into cross - sectional images (2D) thus favoring diagnosis and evaluation. Utilizing this software, D'haese et al stressed the importance of evaluating the alveolar ridges to analyze computerized tomography axial slices ${ }^{1}$.

In 1992 "viewing wand" a frameless system was developed for neurosurgery which served as an adjunct to $\mathrm{CT}^{2}$. Three dimensional imaging was used for presurgical planning and guiding surgeries ${ }^{3,4,5}$.

In the late 1997 dynamic navigation was introduced for oral maxilla-facial surgical procedures on the basis of contribution done by Enisilidis G, Wagner $G$ and Ploder $\mathrm{O}^{6}$. In 2000 VISIT a new dynamic implant navigation system was introduced for implant placement ${ }^{2}$.

\section{COMPUTER-ASSISTED SURGERY}

It involves either a static or a dynamic system. The static system utilizes pre-fabricated stent by using computer aided approaches which can't make real time changes during the implant surgery. Deviations from the pre-determined plan can be carried out using the dynamic systems ${ }^{7}$. The operator need not withdraw from the predetermined plan if they desire to change it during midsurgery in navigation surgery ${ }^{8}$ (Fig 1).

\section{STEPS INVOLVED IN A COMPUTER AIDED}

\section{SURGERY:}

- Cone Beam Computed Tomography ScanningCBCT scanning,

- Software program execution

- Fabrication of surgical drilling guides in case of static approach or a computerized virtual plan in dynamic approach.

- Surgical execution ${ }^{9}$

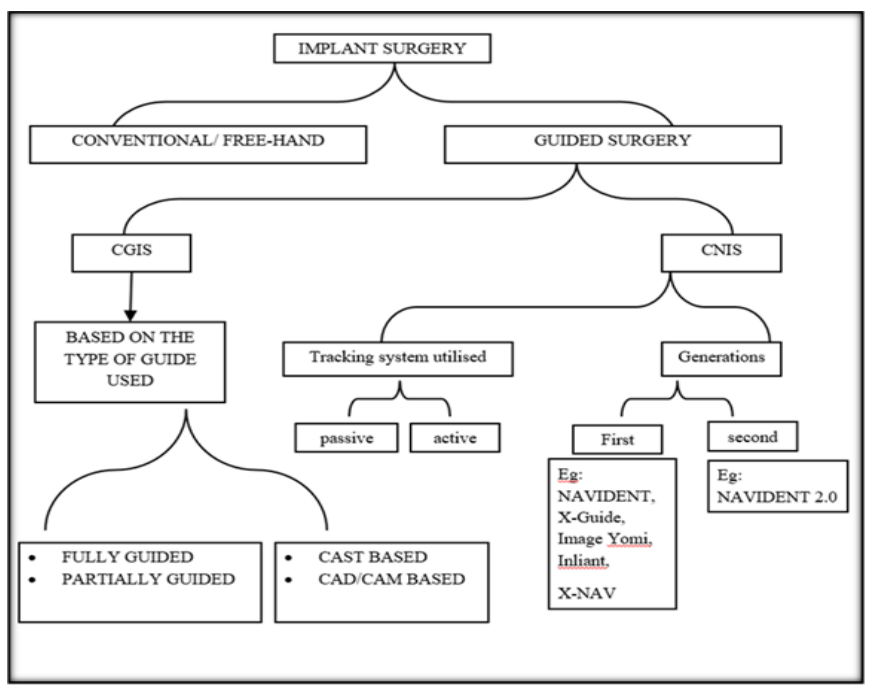

Fig 1: Flow Chart depicting Computer Assisted Implant Surgery

\section{CONVENTIONAL VS COMPUTED AIDED}

\section{APPROACH:}

The implant placement in a free-handed approach is done based on the adjacent and opposing teeth as a reference. In case of computer- assisted surgery, in a static system, CT-generated CAD/CAM stents with metal tubes are used.

In dynamic navigation, a computerized system is used to visualize implant site development while the drills are in function. "Real-time" changes to the plan can be made at the time of surgery ${ }^{3}$.

\section{DYNAMIC NAVIGATION:}

The navigation system consists of stereovision with natural light cameras which utilizes active or passive arrays of optical technologies. Active system utilizes their own light system while the passive systems utilize the light from stereo cameras and the reflected light in order to get tracked for viewing the surgical procedure.

\section{INDICATIONS:}

(i) It is indicated in patients having limited mouth opening with no accessibility.

(ii) It is useful at regions of tight interdental spaces where static guides can't be used owing to the tube size

(iii) (iii) in regions adjacent to the natural teeth, in situations where static guide tubes will interfere with ideal implant placement ${ }^{10,11,12}$. 


\section{COMPONENTS:}

1) Hand piece attachment: It consists of the drill or the handpiece with the drill tag. The drill tag helps to locate the drill position.

2) Patient jaw attachment: It consist of the stent, handle, fiducial marker, fiducial clip an array and a tag. The fiducial marker is the one that enables the whole jaw attachment to be tracked through their identification in CBCT images and clip will help it to be attached to the tag.

3) System cart: It consists of a computer with dynamic navigation software, the stereo cameras with the light source $^{12}$. (Fig 2)

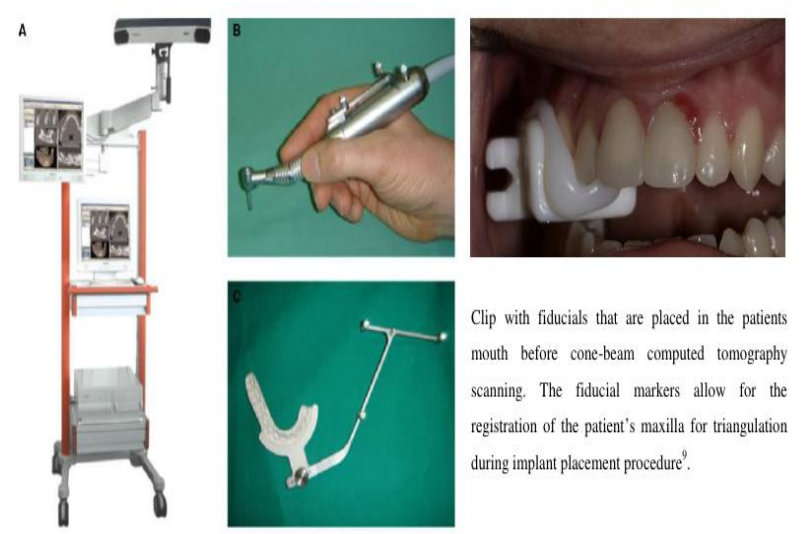

Fig 2: Components of Dynamic navigation (11)

\section{GENERATIONS OF DYNAMIC NAVIGATION}

\section{SYSTEM:}

Based on literature evidence there are two different generations of dynamic navigation. The first generation involved four distinct workflow steps such as stent, scan, plan and place. Commercially available system includes NAVIDENT, X-Guide, Image Yomi, Inliant, X-NAV. The drawbacks of first generation system included high system price, immaturity of design and limited access to CT scanner (Block 2016, Vercruyssen 2014). With the various improvements in the computing and optical tracking technology evolved with elimination of the fiducial clip preparation ${ }^{13}$ and implementation of simpler workflow called TAP - Trace and Place. Commercially available second generation system include NAVIDENT 2.0 .

\section{DYNAMIC WORKFLOW}

\section{FIRST GENERATION SYSTEM}

\section{Stent:}

A thermoplastic retainer molded over the dentition, cured, removed and finished to give guidance to the planned implant placement. Utilizing this, positional approximation is verified and surgery is performed ${ }^{5}$.
Scan

CT marker, thermoplastic stent, retainer arm with fix plate apparatus are secured. Thereafter a single jaw is scanned using a CBCT scanner and the procedure is carried out.

Plan:

Utilizing the scanned jaw image the implant planning is virtually done through placing a computerized implant simulation onto the intended site and alterations can be done.

Place:

The jaw tag is utilized in relation to thermoplastic stent and drill tag. Osteotomy is then performed. A real time "cross-hair" or "bulls-eye" target view is used and osteotomy is completed and implant placement is done under simultaneous tracking likewise.

\section{Steps involved in drill navigation:}

- Initially the procedure starts with mapping of fiducial and extra-oral clip to the CT-image. By doing this the patient and his jaw position are fed to the navigation software.

- Similarly, the drill attachment and its information are further fed to the computer.

- Then the drill tip calibration and drill axis calibration are done. Initially the go plate is registered and calibrated and following that the drill tip is placed for its calibration.

- Following these procedures, the position of the patient's jaw and the drill attachment relative position to each other are constantly tracked through the stereo cameras and fed onto the navigation software.

\section{SECOND GENERATION SYSTEM}

The implant placement protocol is further simplified with the introduction of trace registration. So, the recent protocol is, Trace and Place.

\section{Trace registration:}

In an attempt to achieve ease of operation in the whole dynamic navigation procedure, various technologies have been utilized to carry out implant surgery. Trece registration will eliminate the need for stent fabrication or even a fiducial clip preparation is not needed. Trace registration will trace the recognizable structures naturally present in the scan like teeth, implants, abutments, bony ridge and bone screws as fiducials $^{14}$.

Trace and Place - TAP: 
It is mainly introduced with NAVIDENT 2.0 systems. The CBCT image in DICOM format is installed into dynamic navigation system software (NAVIDENT software). The intra oral scanner image with the virtual wax-up prosthesis is installed and these two images are superimposed and landmarks we marked. Then the trackers have to be attached onto the patient firmly without movements. Then the tracing begins by moving the ball tip of stylus on each tooth for a distance of atleast $15 \mathrm{cms}$. The progression of tracing is indicated audibly and registration completed. For placing implants then drill axis and drill tip calibration are done. The drilling is carried out following the target view on the monitor. It eliminates the need for multiple scans. The implant planning, trace registration and placement all can be completed in single visit. Provided, the implant itself was inserted under guidance EvaluNav, a software evaluates the accuracy and enables to further separate guidance deviation (system error) from drilling deviation (user error) ${ }^{14}$. (Fig 3 )

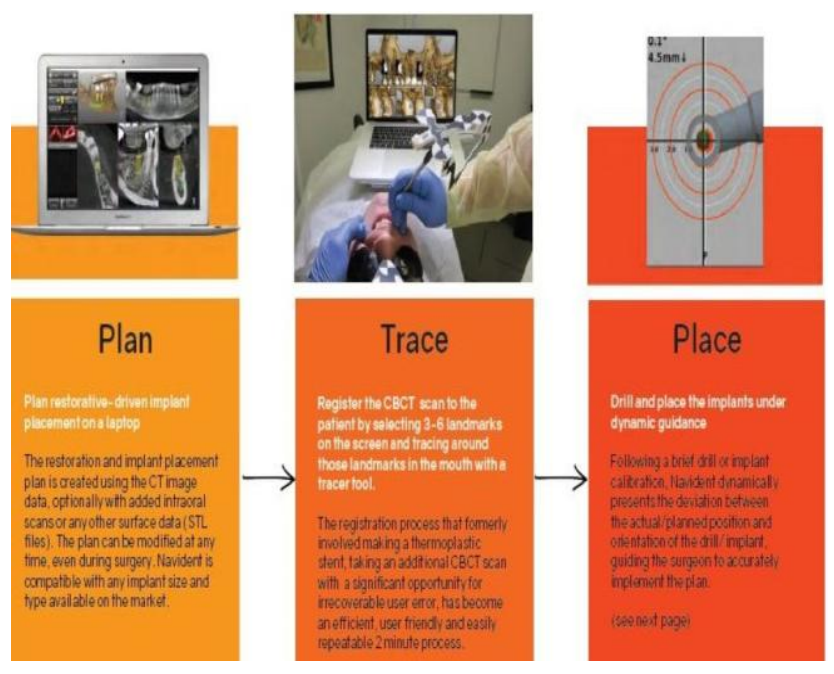

Fig 3: Workflow of trace registration

\section{ADVANTAGES OF DYNAMIC NAVIGATION}

1) In case of complex cases with challenging bone topography and critical anatomic situation dynamic navigation provides its assistance. Wittwer et al in his study supported the accuracy, safer and immediate implant placement in smoother and wide bone areas. In patients with physiological and psychological problems, Vercruyssen et al pointed out that sleeves used as the guide for static approach has rigidity and control in drilling in irregular bony areas.

2) Navigation technology exhibits greater accuracy in recording the coronal and apical portion of implants as cited by few studies which indicated a negligible error of $0.4 \mathrm{~mm}$ of linear deviation and $4^{\circ}$ of angular deviation. This system reported higher precision between planned and finally placed implants 9 .

\section{DISADVANTAGES OF DYNAMIC} NAVIGATION:

(i) Using dynamic navigation systems, when problems arise due to asymmetric bone density may create problems they cannot be avoided.

(ii) Array positions can affect the operation of surgeons' dominant hand in certain cases.

(iii) The reclined or sitting position of patient might affect the cameras' ability to track drill arrays.

(iv) Surgeon's ability to hold the instrument and the rigidity of the instrumentation can interfere with the results. To avoid such short coming the inherent level of tremor of every operator's hand has to be managed with double finger rests $\mathrm{s}^{3,10,12,14}$.

\section{ERRORS IN DYNAMIC NAVIGATION}

The following errors may arise during dynamic navigation such as stent distortion, inaccurate calibration, patient movement, unstable seating of the jaw attachment, operators' eye coordination and lack of personal fine motor control and operators' awareness of the computer technology and systems. Few studies reported accuracy of implants with a mean error of $0.35 \mathrm{~mm}^{9,15,16}$.

\section{APPLICATIONS IN OTHER MEDICAL FIELDS}

It is basically used in surgical decision making and also in execution of surgeries. It locates the anatomic landmarks with precision and ease. It is employed in various neurosurgeries for precise localization and avoidance of areas of risk. It is also used in spinal surgeries and in orthopaedic surgeries as a precise measurement tool ${ }^{17}$.

\section{APPLICATIONS IN OTHER DENTAL FIELDS}

In endodontic, dynamic navigation systems can be used in preparation of minimally invasive access cavity preparations in endodontic. They are also called as conservative/contracted endodontic cavities or ultraconservative "ninja" endodontic cavities and Point endodontic access cavity (PEAC) preparation.

They can be used in identifying and exactly locating calcified canals while performing procedures like root canal treatments ${ }^{19,20}$. It can be utilized to perform endodontic microsurgeries and will greatly help us to create bone cavities and do root end resections and various other procedures. In maxillofacial surgeries computer guided navigation of bone segments are utilized and employed in surgical procedures of TMJ, orthognathic and implant procedures ${ }^{21}$. In maxillo-facial 
Balaji et al: Computer Assisted Dynamic Navigation Systems-Current Scenario

surgery, it is also employed in surgery, Temporomandibular joint surgeries, orthognathic surgeries and Implantology.

\section{CONCLUSION:}

The 3-D diagnostic and therapeutic modalities allow us to overcome various difficulties encountered in case of conventional approaches. It allows the implant team to overcome the difficulties and limitations of human stereo vision and increase the accuracy and precision of implant placement. CAS has its own difficulties like in performing surgery in cost effective fashion. The recurring cost after the initial setting up of these instrument is however low. The learning curve is also another difficulty in performing such procedures. The benefits of these systems also have to be further evaluated to arrive at confirming results. Very few studies are available using this dynamic approach. In future, longitudinal studies are needed in using dynamic navigation system.

\section{FINANCIAL SUPPORT AND SPONSORSHIP}

Nil

\section{CONFLICTS OF INTEREST}

There are no conflicts of interest.

\section{REFERENCES:}

1. D'haese J, Ackhurst J, Wismeijer D, De Bruyn H, Tahmaseb A. Current state of the art of computer-guided implant surgery. Periodontol 2000 2017;73:121-33.

2. Wolfgang Birkfellner, Klaus Huber, Alan Larson, Dennis Hanson, Markus Diemling, Peter Homolka, and Helmar Bergmann. A Modular Software System for Computer-Aided Surgery and Its First Application in Oral Implantology. IEEE Trans Med Imaging, 2000;19(6):616-20.

3. Michael S Block, Robert W Emery, Kathyrn Lank, James Ryan; Implant placement accuracy using Dynamic Navigation. Int $\mathbf{J}$ Oral Maxillofac Implants 2017;32:92-99.

4. Mallaya SM, White SC. The nature of ionizing radiation and risks from maxillofacial cone beam computed tomography. In: Sarment D, ed. Cone Beam Computed Tomography: Oral and Maxillofacial Diagnosis and Applications. Hoboken, NJ: John Wiley \& Sons, Inc; 2014:25-41.

5. Manderalis GA, Stefanelli LV, Degroot BS. Dynamic navigation for surgical implant placement: overview of technology, key concepts, and a case report. Compend Contin Educ Dent 2018.

6. G. Enislidis, A. Wagner, 0. Ploder, R. Ewers. Computed intraoperative navigation guidance-a preliminary report on a new technique. $\mathrm{Br}$ J Oral Maxillofac Surg 1997;35:271-74.

7. Heinz-Theo Luebbers, Peter Messmer, Joachim Anton Obwegeser, Roger Arthur Zwahlen, Ron Kikinis, Klaus Wilhelm Graetz, Felix Matthews. Comparison of different registration methods for surgical navigation in cranio-maxillo-facial surgery. J Craniomaxillofac surg 2008;36:109-16.

8. Jayaranthe YS, Zwahlen RA, Lo J, Tam SC, Cheung LK, Computer aided maxillofacial surgery. Br J Oral Maxillofac surg 2005;43:265-70.

9. Kalaivani G, Balaji VR, Manikandan D, Rohini G. Expectation and reality of guided implant surgery protocol using computer-assisted static and dynamic navigation system at present scenario: Evidence-based literature review. J Indian Soc Periodontol 2020;24:398-408.

10. Michael S Block, Robert W Emery; Static or Dynamic Navigation for Implant PlacementChoosing the Method of Guidance. J Oral Maxillofac Surg 2016;74(2):269-77.

11. Sebastiani FR, Dym H, Emery RW; Dynamic Navigation IN Dental Implant Placement: An In Vivo, Ex Vivo, And In Vitro Systematic Review. Oral Surg Oral Med Oral Pathol Oral Radiol 2017;123(2):e27.

12. Amit Benjamin, Nawamee Phadnis; Dynamic Implant Navigation Systems: A Review. World J Adv Sci Res 2018;1(2):117-22.

13. Chong BS, Dhesi M, Makdissi J. Computer-aided dynamic navigation: a novel method for guided endodontics. Quintessence Int 2019;50:196-202.

14. Stefanelli LV, sMandelaris GA, DeGroot BS, Gambarini G, De Angelis F, Di Carlo S. Accuracy of a Novel Trace-Registration Method for Dynamic Navigation Surgery. Int $\mathbf{J}$ Periodontics Restorative Dent 2020;40(3):427-35.

15. Casap N, Wexler A, Persky N, Schneider A, Lustmann J. Navigation surgery for dental implants: Assessment of accuracy of the image guided implantology system. J Oral Maxillofac Surg 2004;62: 116-9.

16. Emery RW, Merritt SA, Lank K, Gibbs JD. Accuracy of dynamic navigation for dental implant placement-model-based evaluation. J Oral Implantol 2016;42: 399-405.

17. Uli Mezger \& Claudia Jendrewski \& Michael Bartels. Navigation in surgery. Langenbecks Arch Surg 2013;398:501-14.

18. Gianluca Gambarini, Massimo Galli, Antonio Morese, Luigi Vito Stefanelli, Fouad Abduljabbar, Massimo Giovarruscio, Dario Di Nardo, Marco Seracchiani, Luca Testarelli. Precision of dynamic navigation to perform endodontic 
Balaji et al: Computer Assisted Dynamic Navigation Systems-Current Scenario

ultraconservative access cavities: a preliminary in vitro analysis [published online ahead of print, 2020 Jun 15]. J Endod 2020;S0099-2399(20)30385-X.

19. Jain SD, Carrico CK, Bermanis I, Rehil S. Intraosseous Anesthesia using Dynamic NavigationTechnology. [published online ahead of print] J Endod 2020.

20. Saunders MW, Jain SD, Carrico CK, Jadhav A, Deeb JG. Dynamically Navigated versus Freehand
Access Cavity Preparation: A Comparative Study on Substance Loss using Simulated Calcified Canals [published online ahead of print, 2020 Aug 11]. J Endod 2020; S0099-2399(20)30578-1.

21. Rüdiger Marmulla, Herbert Nieder Dellmann. Computer-assisted bone segment navigation. J CranioMaxillofac Surg 1998;26: 347-59. 